\title{
Preparation and characterization of polysulfone membrane coated with poly(ether block amide) for oxygen enrichment process
}

\author{
Kok Chung Chong ${ }^{a,}{ }^{*}$, Yin Yin Chan ${ }^{a}$, Woei Jye Lau ${ }^{b}$, Soon Onn Lai ${ }^{a}$, Ahmad Fauzi Ismail ${ }^{b}$, Hui \\ Shan Thiam ${ }^{\text {a }}$ \\ a Universiti Tunku Abdul Rahman, Lee Kong Chian Faculty of Engineering and Science, Jalan Sungai Long, Bandar Sungai Long, 43300 Kajang, \\ Malaysia \\ b Advanced Membrane Technology Research Centre (AMTEC), Universiti Teknologi Malaysia, 81310 Skudai, Johor, Malaysia \\ * Corresponding author: chongkc@utar.edu.my
}

\section{Article history}

Received 5 May 2018

Revised 1 June 2018

Accepted 2 July 2018

Published Online 4 February 2018

\begin{abstract}
Oxygen enriched air (OEA) is widely applied in various areas such as chemical and medical applications. Currently, cryogenic distillation and pressure swing adsorption are the two common technologies that being commercially used for the production of OEA. However, these two techniques are not economically favorable due to required intensive energy and large built-up area. With the advancement of membrane technology in separation process, it garners the interest from both industrial and academic to explore the feasibility of membrane in gas separation. In this study, polysulfone (PSF) hollow fiber membranes with poly(ether block amide) (PEBAX) coating were used for the separation of $\mathrm{O}_{2} / \mathrm{N}_{2}$ gas. The hollow fiber membranes used in this work were fabricated by phase inversion spinning process using PSF pellet, along with $\mathrm{N}, \mathrm{N}$ dimetyhlacetamide (DMAc) and ethanol $(\mathrm{EtOH})$ as solvent and co-solvent, whereas tetrahydrofuran (THF) as additive. The fabricated membrane exhibited dense structure in the inner layer whereas finger like layer at the outer surface. The formation of this structure was attributed by rapid phase inversion of the solution arose from strong solvent used. The EDX surface mapping analysis confirmed the formation of PEBAX coating on the membrane surface. Gas permeation study in this work illustrated that the pristine PSF membrane exhibited better gas separation performance relative to the PEBAX coated membrane with $20 \%$ higher in terms of permeance. The results obtained from this work suggested that the PEBAX coating enhanced the membrane surface but not certain to improve the gas separation performance. Further study on the PEBAX materials for the membrane coating is essential to polish its potential in gas separation.
\end{abstract}

Keywords: Polysulfone, PEBAX, oxygen, nitrogen, gas separation

\section{INTRODUCTION}

Oxygen enriched air (OEA) is widely used in various fields such as commercial, chemical and medical fields. OEA can be used for sewage treatment, enhancement combustion process and relatively novel application on hybrid system to capture carbon (Belaissaoui et al., 2014). An increase in pure oxygen cost is one of the factors that contributes to the elevated demand on OEA in controlling the emission of combustion or gasification (Chong et al., 2017). Current industrial technologies involved in gas separation are PSA and cryogenic distillation. In the production of OEA, cryogenic distillation can yield the product with above $99 \%$ purity of $\mathrm{O}_{2}$ in large scale production which may exceed 100 tons/day while OEA manufactured by PSA is able to attain oxygen purity at about $95 \%$ with medium production capacity ranging from 20-100 tons/day. However, both technologies are not economically sound and consume high amount of energy (Belaissaoui et al., 2014). Besides, amine solvent adsorption which is an alternative commercial technique to cryogenic distillation in carbon capture is costly and requires regular recharging (Anderson, Wang and Lin, 2012).

Attributed to all these drawbacks rooted from the current available technologies, researchers and industrial players pay their attention on the membrane technology. Membrane technology is regarded as a potential technique that can replace current industrial gas separation techniques. This is due to the fact that membrane system is a relatively smaller in size as compared to traditional systems, mechanically simple and highly flexible enabling it to be scaled up easily (Bernardo and Clarizia, 2013). In addition, polymeric membrane is of interest as it is low cost and ease to process (Kim and Lee, 2013).

Despite of the membrane technology possesses advantages as stated above; its application in the industry of gas separation is still narrow as membrane technology is only able to cater for small scale production (25 tons/day) and to produce lower gas purity as reported by Belaissaoui et al. (2014). Hence, this research wasis mainly focused on the evaluation of performance of polymeric membrane in gas separation by using different amounts of dope solution and also characterization of membrane.

In order to be competitive with other traditional gas separation techniques, a polymeric membrane should possess three main properties which are the permeability of gases involved in gas separation, selectivity towards desired product and good mechanical and thermal characteristics (Powell and Qiao, 2006). The specific membrane material is the factor that affects the flux and selectivity of gases moving across the membrane since different polymers may consist of distinct cross-linkage, morphology and porosity (Smith and Klosek, 2001). 
In this study, the coating of PEBAX material on the surface of self-fabricated membrane was evaluated in terms of its effect on the $\mathrm{O}_{2} / \mathrm{N}_{2}$ gas separation performance. Few membrane characteristics studies were performed to study the properties of the membrane in term of morphology, cross section and the quality of coating on the surface. The gas separation performance of $\mathrm{O}_{2} / \mathrm{N}_{2}$ separation was evaluated by gas permeation study.

\section{EXPERIMENTAL}

\section{Materials}

The polymer used in this study was polysulfone, which obtained in pellet form from Amoco Chemicals. The solvent used to prepare dope solution was $\mathrm{N}, \mathrm{N}$-dimetyhlacetamide (DMAc) with purity more than $99.5 \%$ while ethanol $(\mathrm{EtOH})$ with purity more than $99 \%$ was used as co-solvent, both were obtained from Merck. Tetrahydrofuran (THF) with more than $99.5 \%$ purity from $\mathrm{QReC}$ was added into the dope solution as an additive for solubility enhancement of PSF in the solvent. In this study, polyether-block-amide (PEBAX) obtained from Arkema was employed as membrane coating material. The purpose of PEBAX coating on the membrane surface was to improve the performance of membrane in gas separation by forming a better selective layer on the membrane outer surface.

\section{Preparation of dope solution}

The membrane dope solution composition used in this study was detailed and presented in Table 1. The PSF pellets were placed in the vacuum oven for $24 \mathrm{~h}$ at $70^{\circ} \mathrm{C}$ to remove its moisture content before usage. The dried PSF pellets were mixed with DMAc, EtOH and THF at the predefined ratio to obtain the dope solution. The mixture was stirred for $24 \mathrm{~h}$ with continuous stirring and heating at $60^{\circ} \mathrm{C}$ until a homogeneous dope solution was attained. The dope solution was then undergone a sonication process in an ultrasonic bath for degassing to completely remove the bubbles trapped in the solution.

Table 1 Composition of dope solution.

\begin{tabular}{cc}
\hline Materials & Weight percentage (wt\%) \\
\hline PSF & 30 \\
DMAC & 30 \\
EtOH & 10 \\
THF & 30 \\
\hline
\end{tabular}

\section{Fabrication of hollow fiber membrane}

Phase inversion process was employed to prepare the polysulfone hollow fiber membrane in this work. The dope solution was poured into a spin reservoir and then conveyed to a spinneret with the aid of gear pump. The outer and inner diameters of spinneret were $0.6 \mathrm{~mm}$ and $0.3 \mathrm{~mm}$, respectively. The dope solution and bore liquid were flowed out from the spinneret through the annular space and center, respectively as illustrated in Fig. 1 . The bore liquid flow rate was adjusted at $1.0 \mathrm{~mL} / \mathrm{min}$.

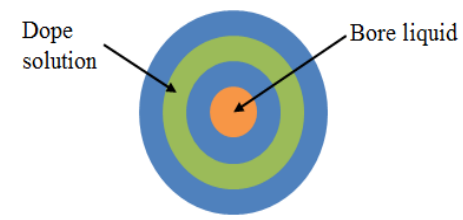

(a)

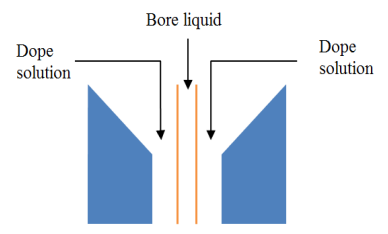

(b)

Fig. 1 The view of spinneret in term of (a) the top (b) cross-section.
The hollow fiber membranes were then delivered to a coagulation bath that filled with tap water at $25^{\circ} \mathrm{C}$. The immersion of membrane in water was carried out for one to two days prior to the drying process in a vacuum oven in order to remove the solvent residues. The hollow fiber membranes were then dried in the vacuum oven at $70^{\circ} \mathrm{C}$ for 24 hours to completely remove the moisture content before usage in the experimental studies. The information of spinning conditions in the phase inversion process was tabulated in Table 2.

Table 2 Spinning conditions of hollow fiber membrane by phase inversion process.

\begin{tabular}{lc}
\hline \multicolumn{1}{c}{ Condition } & Value \\
\hline Bore fluid type & Distilled water \\
Bore fluid temperature $\left({ }^{\circ} \mathrm{C}\right)$ & 25 \\
Bore fluid flow rate $(\mathrm{mL} / \mathrm{min})$ & 1.0 \\
External coagulant type & Tap water \\
External coagulant temperature $\left({ }^{\circ} \mathrm{C}\right)$ & 25 \\
Spinneret dimension OD/ID $(\mathrm{mm} / \mathrm{mm})$ & $0.6 / 0.3$ \\
Air gap distance $(\mathrm{cm})$ & 30 \\
Room relative humidity $(\%)$ & $55 \pm 5$ \\
\hline
\end{tabular}

\section{Preparation of hollow fiber membrane coating}

PEBAX coating solutions were consisted of three different PEBAX concentrations at $1 \mathrm{wt} \%, 3 \mathrm{wt} \%$ and $5 \mathrm{wt} \%$, which then were labelled as PSF-1, PSF-3 and PSF-5, respectively. The hollow fiber membranes utilized in this study were tabulated in Table 3 for ease of reference. The coating solution was prepared by dissolving PEBAX pellet in a mixture of solvent that consisted of ethanol/water at a ratio of 70/30 with the aid of hot plate stirrer at the temperature of $75^{\circ} \mathrm{C}$. The mixture was stirred until a homogenous solution was is obtained. Dip-coating method was employed to coat the fabricated hollow fiber membranes with PEBAX solution. During the coating process, 100 mL PEBAX solution was first transferred to a measuring cylinder and then the membranes prepared were dipped in the solution for $10 \mathrm{~min}$. Later, the hollow fiber membranes were cured in the vacuum oven at $50^{\circ} \mathrm{C}$ for 6 hours. The coating procedure was repeated five times in order to obtain a perfect coating.

Table 3 Hollow fiber membrane engaged in gas permeation study.

\begin{tabular}{cl}
\hline Membrane & \multicolumn{1}{c}{ Description } \\
\hline PSF & Pristine membrane \\
PSF-1 & Membrane with 1 wt\% PEBAX coating \\
PSF-3 & Membrane with 3 wt\% PEBAX coating \\
PSF-5 & Membrane with 5 wt\% PEBAX coating \\
\hline
\end{tabular}

\section{Membrane characterization study}

In this study, scanning electron microscopic (SEM, Hitachi, $\mathrm{S} 3400 \mathrm{~N}$ ) was used to examine the morphology of fabricated hollow fiber membranes. In order to obtain clear SEM images with a perfectcut structure, pre-treatment was carried where the membranes were immersed in liquid nitrogen and broken cryogenically. Later, the membranes were coated with a layer of gold onto the surface by using sputter coating machine (Emitech, SC7620). Additionally, the coating quality of PEBAX on membrane surface was examined by energy dispersive X-ray spectrometer (EDX) under an acceleration voltage of $20 \mathrm{kV}$.

\section{Gas permeation study}

Gas permeation study of the PSF hollow fiber membranes was schematically illustrated in Fig. 2 using pure $\mathrm{N}_{2}$ and $\mathrm{O}_{2}$ gas with purity more than $99.99 \%$. Five hollow fiber membranes were arranged together as a bundle with length of $25 \mathrm{~cm}$, before being inserted into a membrane module and sealed firmly at one end by epoxy resin adhesive. The soap-bubble flow meter consisted of two inlets in which one inlet was connected to the module whereas the other inlet was linked to soap container. During the gas permeation study, pure gas was flowed into the module through the shell side driven by the gas 
pressure at 5 bar. The gas was diffused across the membranes and flowed out from the lumen side of the module where the flow rate was recorded by the soap-bubble flow meter.
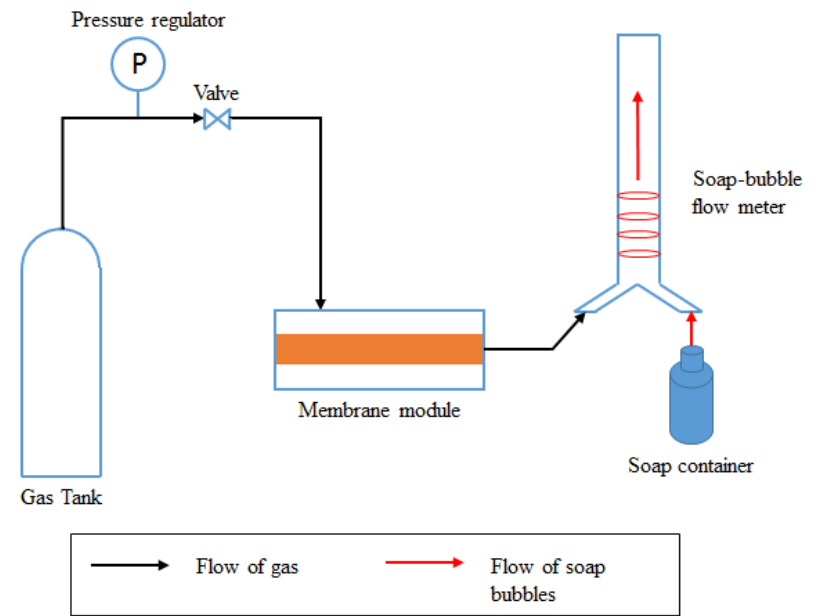

Fig. 2 Schematic diagram of gas permeation study.

Membrane permeability can be defined as the diffusion of gas across the membrane induced by the transmembrane pressure difference. It can be calculated by the following equation,

$$
P_{A}=\frac{Q l}{A \Delta P} \frac{273.15 \times 10^{6}}{T}
$$

where $P_{A}$ is the membrane permeability, $Q$ is the volumetric flowrate of gas $\left(\mathrm{cm}^{3} \mathrm{~s}^{-1}, \mathrm{STP}\right), A$ is the effective membrane area in the unit of $\mathrm{cm}^{2}, \Delta P$ is the pressure difference across the membrane in the unit of $\mathrm{cmHg}, T$ is the operating temperature that is kept constant at $300 \mathrm{~K}$. The unit of the permeability is usually represented as Barrer (1 Barrer $\left.=10^{-10} \mathrm{~cm}^{3}(\mathrm{STP}) \mathrm{cm} / \mathrm{cm}^{2} \mathrm{~s} \mathrm{~cm} \mathrm{Hg}=3.35 \times 10^{-16} \mathrm{~mol} \mathrm{~m} / \mathrm{m}^{2} \mathrm{~s} \mathrm{~Pa}\right)$.

Membrane permeance (GPU or $10^{-6} \mathrm{~cm}^{3}(\mathrm{STP}) / \mathrm{cm}^{2} \mathrm{~cm} \mathrm{Hg}$ ) is defined as the permeability $\left(P_{A}\right)$ per unit length $(l)$ of membrane which can be determined using Eq. 2 :

$$
\frac{P_{A}}{l}=\frac{Q}{A \Delta P} \frac{273.15 \times 10^{6}}{T}
$$

Other than permeance and permeability, selectivity is another important parameter that evaluates the performance of membrane. The selectivity of binary gas separation $\left(\alpha_{A / B}\right)$ is represented by the ratio of the permeability of gas $\mathrm{A}\left(P_{A}\right)$ to the permeability of gas $\mathrm{B}\left(P_{B}\right)$ can be determined by Eq. 3:

$$
\alpha_{\mathrm{A} / \mathrm{B}}=\frac{\mathrm{P}_{\mathrm{A}}}{\mathrm{P}_{\mathrm{B}}}
$$

\section{RESULTS AND DISCUSSION}

\section{Membrane morphology}

The membrane morphology for the hollow fiber membrane coated with PEBAX (PSF-1, PSF-3 and PSF-5) was shown in Fig. 3. All the fabricated hollow fiber membranes illustrated dense structure at the inner layer with the finger-like layer at the outer surface. This structure was attributed by the rapid phase inversion of the solution arose from strong solvent used in this study (Wu et al., 2006). The diameter of the membrane fabricated from this study was consistent which was in the range of 310 to $370 \mu \mathrm{m}$ (Table 4). The characteristics study showed that the concentration of PEBAX was not obviously affect the wall thickness of PSF membrane where the wall thickness of the membrane was in the range of 50 to $54 \mu \mathrm{m}$. The EDX results on the quality of the PEBAX coating on the surface were tabulated in Table 5. Nitrogen was found to be presented only in the
PSF-1, PSF-3 and PSF-5 as nitrogen is the was contained in the amide group in PEBAX. It was interesting to note that the content of nitrogen was increased as the coating concentration of the PEBAX on hollow fiber membrane was increased, showing that the PEBAX was successfully coated on the surface of the membrane.
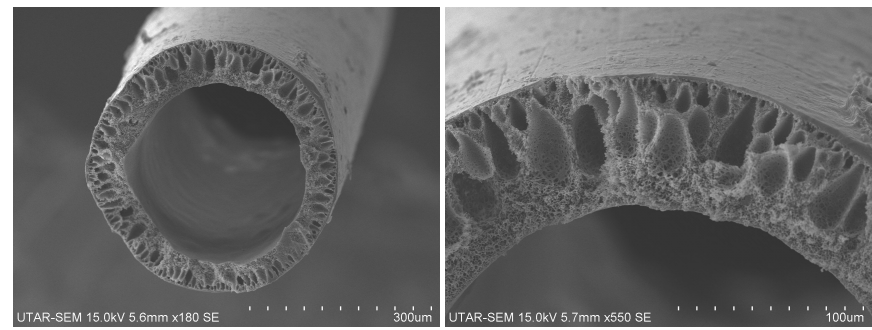

(a)
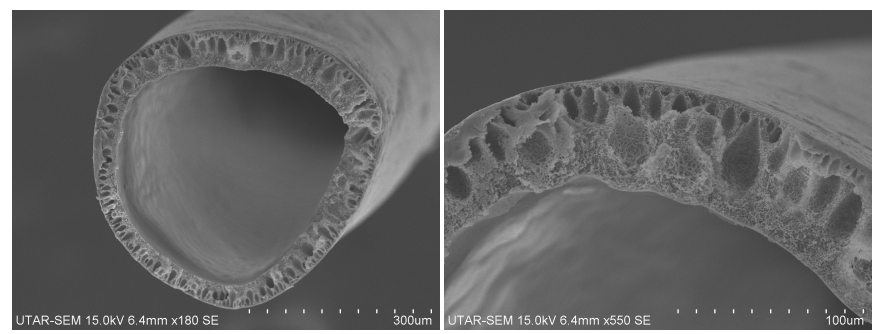

(b)
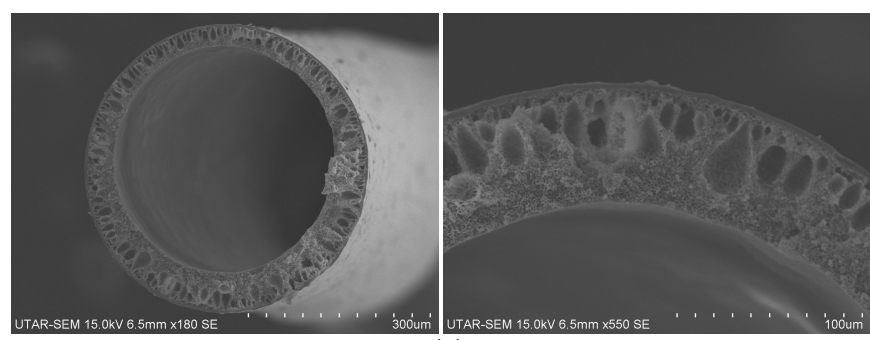

(c)

Fig. 3 Membrane cross sectional morphology of PEBAX coated PSF membrane (a) PSF-1 (b) PSF-2 (c) PSF-3.

Table 4 Membrane characterisrics.

\begin{tabular}{ccc}
\hline Membrane & $\begin{array}{c}\text { Internal diameter } \\
(\boldsymbol{\mu} \mathbf{m})\end{array}$ & $\begin{array}{c}\text { Wall Thickness } \\
(\boldsymbol{\mu m})\end{array}$ \\
\hline & & \\
PSF & $370 \pm 10$ & $50 \pm 4$ \\
PSF-1 & $310 \pm 10$ & $54 \pm 8$ \\
PSF-3 & $350 \pm 5$ & $51 \pm 7$ \\
PSF-5 & $360 \pm 5$ & $50 \pm 7$ \\
& & \\
\hline
\end{tabular}

Table 5 EDX analysis for the membrane.

\begin{tabular}{ccccc}
\hline Membrane & Carbon & Oxygen & Sulphur & Nitrogen \\
\hline PSF & 82.61 & 14.19 & 3.20 & Not detected \\
PSF-1 & 76.00 & 17.82 & 3.08 & 3.10 \\
PSF-3 & 75.61 & 16.70 & 3.01 & 4.68 \\
PSF-5 & 74.44 & 17.54 & 3.09 & 4.93 \\
& & & & \\
\hline
\end{tabular}

\section{Membrane Perfomance in OEA Production}

The gas permeation results in $\mathrm{O}_{2} / \mathrm{N}_{2}$ separation performance by PSF membranes were recorded in Table 6. It was found that both permeability of oxygen and $\mathrm{O}_{2} / \mathrm{N}_{2}$ selectivity were decreased when the concentration of PEBAX on the membrane was increased.The coating of PEBAX solution on the membrane surface was able to improve the smoothness of the membrane surface, nevertherless, it still possessed little advantage in the seperation performance. The results obtained in the gas permeation study were opposed to the literature studies whereby the addition of PEBAX coating on the membrane surface was able to improve the permeability while 
maintaining the selectivity of the gas separation (Wang et al. 2014). However, it was noteworthy to mention that the works done by previous literature reviews were carried out using flat sheet membrane coated with PEBAX solution (Ren et al., 2012, Ahmadpour et al., 2014 and Wang et al. 2014). The lower gas permeation results obtained from this study might attribute to the PEBAX coating as some of the hollow fiber membranes were tended to stick to each other during the curing process. Hence, the additional optimization was required in our future work in order to release the potential of PEBAX coating on the hollow fiber membrane. On the other hand, the performance of pristine PSF membrane was attributed to its superior gas separation peformance which eventually led to higer yield of OEA production.

Table 6 Performance of PSF membrane on $\mathrm{O}_{2} / \mathrm{N}_{2}$ separation.

\begin{tabular}{cccccc}
\hline \multirow{2}{*}{ Membrane } & \multicolumn{2}{c}{$\begin{array}{c}\text { Permeance } \\
\text { (GPU) }\end{array}$} & \multicolumn{2}{c}{$\begin{array}{c}\text { Permeability } \\
\text { (Barrer) }\end{array}$} & \multirow{2}{*}{$\begin{array}{c}\mathbf{O}_{2} / \mathbf{N}_{\mathbf{2}} \\
\text { selectivity }\end{array}$} \\
\cline { 2 - 5 } & $\mathbf{O}_{\mathbf{2}}$ & $\mathbf{N}_{\mathbf{2}}$ & $\mathbf{O}_{\mathbf{2}}$ & $\mathbf{N}_{\mathbf{2}}$ & \\
\hline PSF & 51.00 & 13.00 & 12.75 & 3.25 & 3.92 \\
PSF-1 & 39.81 & 10.73 & 9.95 & 2.68 & 3.71 \\
PSF-3 & 36.15 & 11.52 & 9.04 & 2.88 & 3.14 \\
PSF-5 & 33.28 & 10.14 & 8.32 & 2.54 & 3.28 \\
\hline
\end{tabular}

\section{CONCLUSION}

In this study, PSF hollow fiber membranes were successfully fabricated and coated with PEBAX materials under different weight concentrations. Membrane characterization and gas permeation studies were conducted to understand the properties of the membranes and evaluate the membrane performance in the $\mathrm{O}_{2} / \mathrm{N}_{2}$ gas separation for OEA production. The EDX results indicated that PEBAX was successfully coated on the surface of the membrane with the presence of nitrogen from amide group. Results from gas permeation study suggested that the PEBAX coating exhibited modest advantages relative to the pristine membrane in $\mathrm{O}_{2} / \mathrm{N}_{2}$ gas separation but it was able to enhance the surface of the hollow fiber membrane. The result from this study proposed that PEBAX materials were able to act as membrane surface enhancement and further study on the PEBAX materials on the membrane coating was is essential to release its potential in gas separation.

\section{ACKNOWLEDGEMENT}

This work was financially supported by Mayair Manufacturing (M) Sdn. Bhd. under the Studentship Research Grant (Vote No: 4464/000) and Universiti Teknologi Malaysia for the researchfunding provided under Research University Grant (Vot no: Q.J130000.2546.15H41)

\section{REFERENCES}

Ahmadpour E., Shamsabadi A. A., Behbahani R. M., Aghajani M., Kargari A. (2014). Study of $\mathrm{CO}_{2}$ separation with PVC/Pebax composite membrane. Journal of Natural Gas Science and Engineering, 21, pp.518-523.

Anderson, M., Wang, H., Lin, Y. (2012). Inorganic membranes for carbon dioxide and nitrogen separation. Reviews in Chemical Engineering, 28(23), pp.101-121.

Belaissaoui, B., Le Moullec, Y., Hagi, H., Favre, E. (2014). Energy efficiency of oxygen enriched air production technologies: Cryogenyvs membranes. Separation and Purification Technology, 125, pp.142-150.

Bernardo, P., Clarizia, G. (2013). 30 years of membrane technology for gas separation. Chemical Engineering Transactions, 32, pp.1999-2004.

Chong, K. C. , Lai, S. O., Lau, W. J., Thiam, H. S., Ismail, A., Zulhairun, A (2017). Fabrication and characterization of polysulfone membranes coated with polydimethysiloxane for oxygen enrichment. Aerosol and Air Quality Research, pp. $1-8$.

Chong, K. C., Lai, S. O., Thiam, H. S., Teoh, H. C., Heng S. L. (2016). Recent progress of oxygen/nitrogen separation using membrane technology. Journal of Engineering Science and Technology, 11(7), pp. 1016-1030.

$\mathrm{Kim}$, S., Lee, Y. (2013). High performance polymer membranes for CO2 separation. Current Opinion in Chemical Engineering, 2(2), pp.238-244.

Peng, N., Widjojo, N., Sukitpaneenit, P., Teoh, M., Lipscomb, G., Chung, T., Lai, J. (2012). Evolution of polymeric hollow fibers as sustainable technologies: Past, present, and future. Progress in Polymer Science, 37, pp.1401-1424.

Powell, C., Qiao, G. (2006). Polymeric CO2/N2 gas separation membranes for the capture of carbon dioxide from power plant flue gases. Journal of Membrane Science, 279(1-2), pp.1-49.

Ren, X., Ren, J., Deng, M., (2012). Poly(amide-12-b-ethylene oxide)/polyethylene glycol blend membranes for carbon dioxide separation. Separation and Purification Technology, 89, pp.1-8.

Smith, A., Klosek, J. (2001). A review of air separation technologies and their integration with energy conversion processes. Fuel Processing Technology, 70(2), pp.115-134.

Wang, L., Li, Y., Li, S., Ji, P., Jiang, C., (2014). Preparation of composite poly(ether block amide) membrane for $\mathrm{CO}_{2}$ capture. Journal of Energy Chemistry, 6, pp.717-725.

Wu, L. S., Sun, J. F., Wang, Q. R. (2006). Poly(vinylidene fluoride)/polyethersulfone blend blend membranes: Effects of solvent sort, polyethersulfone and polyvinylpyrrolidone concentration on their properties and morphology. Journal of Membrane Science, 285, pp.290 298. 